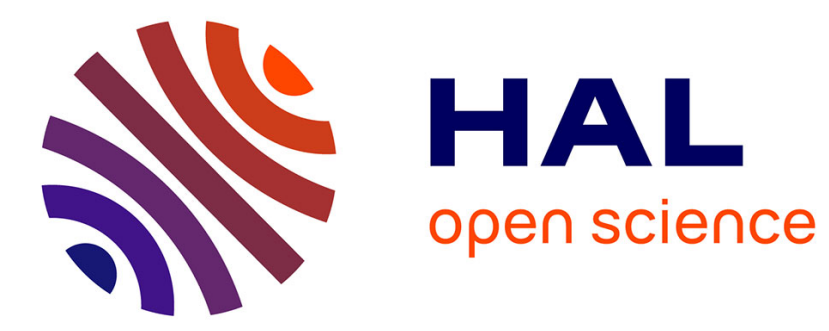

\title{
La materia nei commenti al Timeo del secolo XII Irene Caiazzo
}

\section{To cite this version:}

Irene Caiazzo. La materia nei commenti al Timeo del secolo XII. Quaestio. Annuario di storia della metafisica, 2007, 7, pp.245-264. halshs-00293912v2

\section{HAL Id: halshs-00293912 \\ https://shs.hal.science/halshs-00293912v2}

Submitted on 8 Jul 2008

HAL is a multi-disciplinary open access archive for the deposit and dissemination of scientific research documents, whether they are published or not. The documents may come from teaching and research institutions in France or abroad, or from public or private research centers.
L'archive ouverte pluridisciplinaire HAL, est destinée au dépôt et à la diffusion de documents scientifiques de niveau recherche, publiés ou non, émanant des établissements d'enseignement et de recherche français ou étrangers, des laboratoires publics ou privés. 


\section{Irene Caiazzo}

\section{La materia nei commenti al Timeo del secolo XII}

Due traduzioni latine parziali del Timeo di Platone sono disponibili nel Medioevo: quella effettuata da Cicerone (Tim. 27D-47B, con delle lacune) nel I secolo a.C. e quella di Calcidio (Tim. 17A-53C) da collocare tra il IV e il V secolo della nostra èra. Quest'ultimo scrisse anche un ricco e complesso Commentarius in Timaeum, di ispirazione medio-platonica, il cui piano dettagliato è presentato nel prologo. Ora tale Commentarius non ci è pervenuto integralmente, o meglio, non si sa se Calcidio abbia realmente eseguito l'ambizioso piano iniziale da lui annunciato nel prologo ${ }^{1}$ oppure si sia limitato a esporre alcuni passi scelti del Timeo compresi tra $31 \mathrm{C}$ e $53 \mathrm{C}$, cioè la porzione - e si tratta di una porzione voluminosa - del Commentarius conosciuta sin dal Medioevo, senza dimenticare che frammenti più o meno lunghi del Timeo sono pure citati, e dunque trasmessi in forma indiretta, da autori come Apuleio, Seneca, Macrobio, Agostino, Nemesio di Emesa e tanti altri ${ }^{2}$. Se la traduzione ciceroniana non sembra aver riscosso molto successo nel Medioevo, nonostante se ne conservino diversi manoscritti, la versione di Calcidio fu oggetto di diversi commenti, redatti tutti prevalentemente nel secolo XII.

Gli interpreti moderni del Timeo discutono ancora per capire se in questo dialogo platonico la materia primordiale/hyle, chiamata anche ricettacolo, spazio, luogo, madre, nutrice, sia creata oppure increata e se, eventualmente, la sua

\footnotetext{
I Il piano dell'opera esposto nel prologo prevede ventisette capitoli, ma sono conservati invece solo i primi tredici, e il tredicesimo è intitolato per l'appunto De silva.

2 M. Lemoine, Innovations de Cicéron et de Calcidius dans la traduction du «Timée», in R. ELLIS / R. Tixier / B. Weitemeier (eds.), The Medieval translator. Traduire au Moyen Âge. Actes du Colloque international de Göttingen (22-25 juillet 1996), Brepols, Turnhout 1997, pp. 72-81; ID., Le «Timée» latin en dehors de Calcidius, in A. De Libera / A. Elamrani-Jamal / A. Galonnier (éds.), Langages et philosophie. Hommage à Jean Jolivet, Vrin, Paris 1997, pp. 63-78; ID., Du «Timée» de Platon à celui de Calcidius, in

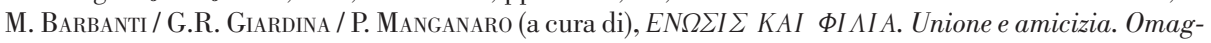
gio a Francesco Romano, Cuecm, Catania 2002, pp. 441-450.
} 
creazione sia da collocare nel tempo o fuori dal tempo ${ }^{3}$. Nella traduzione latina di Calcidio necessitas è il termine più ricorrente per designare la materia primordiale; passando poi dalla traduzione al Commentarius, che sempre fu la guida dei lettori medievali, la situazione si complica e il vocabolario risulta meno uniforme. Il trattato sulla materia primordiale occupa la parte finale ed anche la più estesa del Commentarius, capp. 268-355 dell'edizione Waszink, ed è intitolato De silva; esso comincia con l'esame del lemma del Timeo 47E: Nunc quoniam cuncta exceptis admodum paucis executi sumus, che demarca una divisione importante nel dialogo platonico, perché dopo aver parlato delle cose realizzate dall'intelletto della mente provvidenziale (providae mentis intellectus) si passa alle cose che provengono dalla necessità. Calcidio spiega che Platone ha designato la materia primordiale con il termine necessitas, riferendosi ovviamente alla sua traduzione latina, ma che i latini possono chiamarla silva, termine questo di chiara fortuna tra gli autori medievali:

«Necessitatem porro nunc appellat hylen, quam nos latine silvam possumus nominare, ex qua est rerum universitas eademque patibilis natura, quippe subiecta corpori principaliter, in qua qualitates et quantitates et omnia quae accidunt proveniunt; quae cum a natura propria non recedat, diversis tamen et contrariis speciebus eorum quae intra se recipit formisque variatur ${ }^{4}$.

Il mondo sensibile è il risultato della mescolanza della provvidenza e della necessità, nel senso che il mondo nasce dalla deliberazione della provvidenza e dalle cause della necessità, cioè la provvidenza opera e agisce sulla silva che si lascia plasmare e penetrare dalla provvidenza ${ }^{5}$. Necessitas dunque non perché la causa principale della costituzione del mondo sensibile sia la silva o nella silva ma perché è «necessario» impiegarla per dare una sostanza corporea alle co$\mathrm{se}^{6}$. La materia primordiale, visto che è un principio, non può essere mostrata

3 Tra i tanti contributi pubblicati sulla materia nel Timeo, si citano soltanto i seguenti recenti articoli: J.-M. NARbonne, Le réceptacle platonicien: nature, fonction, contenu, «Dialogue», 36 (1997), pp. 253279; L. Brisson, À quelles conditions peut-on parler de «matière» dans le «Timée» de Platon?, «Revue de métaphysique et de morale» (janvier-mars 2003, nr. 1), pp. 5-21; G.R. CARone, Creation in the "Timaeus»: The Middle Way, «Apeiron», 37/3 (2004), pp. 211-226.

${ }^{4}$ Calcidius, Commentarius 268, in ID., Timaeus a Calcidio translatus commentarioque instructus, ed. J.H. Waszink / P.J. Jensen, The Warburg Institute, London / Brill, Leiden $1975^{2}$ («Plato Latinus», 4), p. 273. Cf. anche la bella traduzione con testo latino a fronte: CAlcidio, Commentario al «Timeo» di Platone, ed. C. Moreschini, Bompiani, Milano 2003.

5 Calcidius, Commentarius 269, ed. Waszink, p. 274: «Silva vero perpetiente exornationique se facilem praebente, penetratam siquidem eam usque quaque divina mens format plene, non ut artes formam tribuentes in sola superficie, sed perinde ut natura atque anima solida corpora permeantes universa vivificant».

${ }^{6}$ Calcidius, Commentarius 270, ed. Waszink, p. 275. 
attraverso degli esempi né spiegata grazie a una causa che la precede, ma è conoscibile soltanto in maniera indiretta dall'intelletto attraverso l'astrazione delle forme in essa contenuta, poiché la materia che si percepisce attraverso i sensi possiede forma, qualità, quantità, ecc., si tratta cioè della materia sensibile in cui sono già entrate le forme e non della materia primordiale. Una volta eliminati tutti i corpi e le qualità contenuti nella silva, si potrà allora immaginare con la speculazione della mente un grembo vuoto (sinus vacuus) ${ }^{7}$. Calcidio si serve di diversi termini per designare la materia primordiale: silva, hyle, necessitas, erratica causa, mater, nutricula, locus, a seconda dell'aspetto o del ruolo della materia primordiale sul quale vuole insistere ${ }^{8}$.

Gli autori medievali che si cimentano nell'arduo compito di commentare il Timeo leggono dunque perlomeno cinque vocaboli latini differenti per indicare la materia primordiale. Siamo oramai ben lontani dal testo greco di Platone: una prima mediazione è effettuata dalla traduzione latina di Calcidio, una seconda mediazione dal Commentarius che complica considerevolmente la questione perché in esso vengono impiegate concezioni totalmente estranee agli autori medievali ignari delle filosofie della Tarda Antichità, per essi quasi interamente inaccessibili. Il Commentarius di Calcidio è un testo difficile ed erudito che non ha finito di svelare tutti i suoi misteri, a lungo trascurato dagli storici della filosofia perché ritenuto minore o ancora peggio «eclettico» e dunque poco interessante $^{9}$. Ma non è questa l'opinione dei commentatori medievali che leggendo e glossando il dialogo platonico spesso utilizzano, senza esserne pienamente consapevoli, concetti di sapore stoico, aristotelico, medio-platonico, neo-platonico provenienti dal Commentarius. Calcidio presenta infatti non solo la sua interpretazione della lettera platonica ma espone anche l'opinione di diverse sette filosofiche sulla materia primordiale. Egli introduce la trattazione affermando che nessuno degli antichi dubitava dell'esistenza della materia primordiale, però alcuni pensavano che fosse fatta (facta) e altri che fosse non generata (sine generatione). Tra i fautori dell'opinione che la materia fosse non generata, 1) alcuni la consideravano continua e uniforme e si chiedevano poi se le forme e le qualità possedute dalle cose in essa contenute provenissero dalla materia stessa 0 da un'altra potenza superiore ed esterna alla materia; 2 ) altri invece concepivano la materia come divisibile in parti e si domandavano se la materia fosse scevra di forma o di qualità oppure, al contrario, se essa fosse dotata di forma propria. Gli Ebrei ritenevano invece che la silva fosse stata generata (generata) $)^{10} \mathrm{e}$

${ }^{7}$ Calcidius, Commentarius 274, ed. Waszink, pp. 278-279.

8 J.R. O'Donnell, The Meaning of «Silva» in the Commentary on the "Timaeus» of Plato by Chalcidius, «Mediaeval Studies», 7 (1945), pp. 1-20; J.C.M. van WInden, Calcidius on Matter. His Doctrine and Sources. A Chapter in the History of Platonism, Brill, Leiden $1965{ }^{2}$. 
che tale generazione fosse avvenuta fuori dal tempo, cioè la silva avrebbe avuto un inizio prima della formazione del mondo e quindi prima del tempo, prima che l'avvicendamento dei giorni e delle notti permettesse di misurare il tempo. A parte gli Ebrei, tutti gli altri avevano dunque asserito che la silva era non generata: gli epicurei, gli stoici, Aristotele, Talete, Anassagora, Platone, i discepoli di Platone. Questi ultimi - spiega Calcidio - non hanno però sempre rispettato l'insegnamento del Timeo; per esempio, alcuni di essi sostengono che secondo Platone la materia è generata, altri poi ritengono che la materia sia sì sine generatione ma dotata di un'anima, dal momento che Platone aveva affermato che vi era un movimento confuso nella materia, prima che questa venisse ordinata dall'artefice ${ }^{11}$. Calcidio presenta infine la sua teoria sulla materia primordiale nella quale si ritrovano diversi elementi caratteristici del platonismo dell'età imperiale: la materia primordiale è un principio, precisamente è uno dei tre principi primi (quibus antiquius nihil est) insieme all'artefice e alle idee. Se la materia può essere compresa attraverso la ragione, grazie a uno dei due modi di procedere della dimostrazione, l'analisi (resolutio), separando cioè dalla materia le qualità, le quantità e le forme, gli altri due principi, l'artefice e le idee, possono essere compresi grazie alla sintesi (compositio), l'altro procedimento della dimostrazione ${ }^{12}$. La materia primordiale è dunque un principio (initium), il primo limite da cui nascono tutte le cose; essa precede cioè la nascita di tutte le cose e in quanto tale è semplice, eterna e priva di qualità:

«Est igitur initium primus limes, post quem sunt cuncta quae ex eodem sunt limite; et quia omne initium, utpote origo, rerum praecedit ortum, necesse est eam fore simplicem sine qualitate perpetuam» ${ }^{13}$.

Alla stregua della cera, la materia primordiale accoglie nel suo seno le forme impresse dall'artefice - o meglio le immagini delle forme intelligibili, come spiegherà Calcidio nei capitoli finali del suo Commentarius $^{14}$-, senza subire alcuna trasformazione nella sua natura (natura è impiegato come sinonimo di sub-

9 Cf. S. Gersh, Calcidius' Theory of First Principles, «Studia Patristica», 18/2 (1989), pp. 85-92; G. Reydams-Schils, Demiurge and Providence. Stoic and Platonist Readings of Plato's «Timaeus», Brepols, Turnhout 1999, pp. 207-243; EAD., «Calcidius Christianus»? God, Body, and Matter, in T. Koвusch / M. Erler / I. Männlein-Robert (Hrsg.), Metaphysik und Religion. Zur Signatur des spätantiken Denkens. Akten des Internationalen Kongresses vom 13-17 März 2001 in Würzburg, Saur, München-Leipzig 2002 («Beiträge zur Alterumskunde», 160), pp. 193-211.

10 Calcidius, Commentarius 276, ed. Waszink, p. 280.

${ }^{11}$ Calcidius, Commentarius 300-301, ed. Waszink, pp. 301-303.

12 Calcidius, Commentarius 303-304, ed. Waszink, pp. 305-306.

13 Calcidius, Commentarius 305, ed. Waszink, p. 306. Relativamente ai principi primi, Calcidio utilizza indifferentemente aeternus e perpetuus.

${ }^{14}$ Calcidius, Commentarius 329, 337, ed. Waszink, pp. 323, 330-331. 
stantia); in più luoghi Calcidio ribadisce che la materia deve essere intesa come il receptaculum delle forme che provengono dall'esterno, criticando apertamente gli stoici i quali ritengono che le forme e le qualità fluiscono dalla materia stessa, rendendo in tal caso superflua e vana l'azione dell'artefice ${ }^{15}$; inoltre la materia è infinita e illimitata (nel caso contrario essa sarebbe dotata di qualità e forma) $)^{16}$, non subisce poi né incremento né diminuzione, non è divisibile, è immobile; i corpi in essa contenuti, in quanto provvisti di quantità e di qualità, potranno essere divisi ed essere mossi ma non la silva che li accoglie. Essa poi non è né corporea - in quanto non ha né forma né qualità e soprattutto non è percepibile dai sensi ${ }^{17}$ - né incorporea - in quanto nessun incorporeo subisce la condizione del corpo o può trasformarsi in corpo. Dunque né sensibile né intelligibile, la silva è un corpo in potenza oppure un non corpo in potenza: «recte igitur eam simpliciter et ex natura sua neque corpoream neque incorpoream cognominamus, sed possibilitate corpus et item possibilitate non corpus» ${ }^{18}$. Infine, Calcidio insiste sul fatto che la silva non deve essere confusa con i quattro elementi ${ }^{19}$ che subiscono trasformazioni reciproche e che non possono essere considerati in alcun caso «principi primi» del mondo.

Il giudizio di J.R. O'Donnell su Calcidio è senza appello: «no clearcut and consistent doctrine of matter emerges from a study of the Commentary of Chalcidius on the Timaeus. Only very general points can be stated with certainty» 20 . Calcidio avrebbe infatti combinato Platone e Aristotele, o meglio avrebbe interpretato Platone servendosi di un bagaglio principalmente aristotelico, e inoltre avrebbe oscillato fra una teoria della materia primordiale intesa in certi passi come un in quo e in altri come un ex quo. Questa stessa esitazione ermeneutica in Calcidio è colta già nel secolo XII da Bernardo di Chartres che, nelle glosse al Timeo a lui recentemente attribuite, indica diverse interpretazioni possibili dei luoghi platonici a seconda che la materia sia concepita come un in quo o come un ex quo. In ogni modo, il lungo capitolo De silva del Commentarius di Cal-

15 Calcidius, Commentarius 311, ed. Waszink, p. 311: «Quare si intra silvam ratio formarum et qualitatum latet, ut Stoicis videtur, abundat opificis moderatio, sed opinor silvae opificem necessarium, sicut ipsi etiam Stoici sanciunt. Opifex igitur silvae ut aeris informitati ceraeve formas, insigniet atque ita constabit ob necessariam dogmatis rationem».

16 Calcidius, Commentarius 312, ed. Waszink, pp. 311-312.

17 Calcidius, Commentarius 319, ed. Waszink, pp. 314-316.

18 Calcidius, Commentarius 320, ed. Waszink, p. 316.

19 Calcidius, Commentarius 316, ed. Waszink, pp. 312-313: «Recta est igitur nostra opinio neque ignem neque terram nec aquam nec spiritum esse silvam, sed materiam principalem et corporis primam subiectionem, in qua non qualitas non forma non quantitas non figura sit ex natura propria, sed virtute opificis haec ei cuncta conexa sint, ut ex his universo corpori et singillatim perfectio et communiter varietas comparetur».

20 O'DonnelL, The Meaning of «Silva» cit., p. 19. 
cidio resta la trattazione più completa e autorevole di tutta la produzione filosofica latina antica e tardo-antica sulla materia primordiale, una miniera di informazioni sulle opinioni dei filosofi greci a cui gli autori medievali attingono considerevolmente, in modo particolare prima delle traduzioni delle opere aristoteliche realizzate nel corso dei secoli XII e XIII. Il ruolo di Calcidio e del Commentarius è stato certo evocato dagli storici della filosofia ma mai studiato con la dovuta attenzione. La questione della materia nei commenti medievali al $T_{i-}$ meo è complessa e può essere studiata da diversi punti di vista: relazione Diomateria, materia-forme intelligibili, materia-mondo sensibile, materia-quattro elementi, materia-anima, materia-tempo ${ }^{21}$. In questa sede, mi limiterò a delle osservazioni di carattere generale sulla materia primordiale prendendo in esame alcuni passi «chiave» del commento al Timeo attribuito a Bernardo di Chartres e del commento di Guglielmo di Conches, lasciando da parte gli altri commenti anonimi ancora inediti del secolo XII22.

\section{Le Glosae super Platonem attribuite a Bernardo di Chartres}

Bernardus quoque Carnotensis perfectissimus inter Platonicos saeculi nostri, così si esprime Giovanni di Salisbury, nel Metalogicon, facendo l'elogio di questo grande maestro chartriano attivo nel primo quarto del secolo XII23. A parte le magre, ambigue e forse non sempre affidabili testimonianze dello stesso Gio-

${ }^{21}$ Sulla questione della materia nel secolo XII si possono consultare: H. FlatTEn, Die «materia primordialis» in der Schule von Chartres, «Archiv für Geschichte der Philosophie», 40 (1931), pp. 58-65; J.M. Parent, La doctrine de la Création dans l'École de Chartres, Institut d'Études Médiévales et Vrin, Paris-Ottawa 1938 («Études et Textes»); T. Gregory, Platonismo medievale. Studi e ricerche, Istituto Storico Italiano per il Medio Evo, Roma 1958; M.-D. CHEnu, La théologie au douzième siècle, Vrin, Paris 19762; J. Moreau, «Opifex, id est Creator». Remarques sur le platonisme de Chartres, «Archiv für Geschichte der Philosophie», 56 (1974), pp. 33-49; R.C. Dales, Marius «On the Elements» and the Twelfth-Century Science of Matter, «Viator», 3 (1972), pp. 191-218; J. JoLIVET, La question de la matière chez Gilbert de Poitiers, in H.J. Westra (ed.), From Athens to Chartres: Neoplatonism and Medieval Thought. Studies in Honour of Édouard Jeauneau, Brill, Leiden-New York-Köln 1992, pp. 247-257.

${ }_{22}$ M. GIBson, The Study of the «Timaeus» in the Eleventh and Twelfth Centuries, «Pensamiento», 25 (1969), pp. 183-194; P.E. DutTon, Material Remains of the Study of the "Timaeus» in the Later Middle Ages, in C. Lafleur / J. Carrier (éds.), L'enseignement de la philosophie au XIIIe siècle. Autour du «Guide de l'étudiant» du ms. Ripoll 109, Brepols, Turnhout 1997, pp. 203-230. Gli altri due commenti «maggiori» del secolo XII sono l'Apparatus super Thimeum conservato nel manoscritto di Salamanca, Biblioteca Universitaria, 2322 e il Super Thimeum Platonis conservato nel manoscritto di Oxford, Bodleian Library, Corpus Christi College 243. Per il commento di Salamanca, si veda l'articolo sopra menzionato di Dutton; cf. anche I. Caiazzo, Sur la distinction sénéchienne «idea/idos» au XIIe siècle, «Chora. Revue d'études anciennes et médiévales», 3-4 (2005-2006), pp. 91-116.

${ }^{23}$ Ioannes SAResberiensis, Metalogicon IV, 35, ed. J.B. Hall / K.S.B. Keats-Rohan, Brepols, Turnhout 1991 («Corpus Christianorum, Continuatio medievalis», 98), p. 173. 
vanni sulle dottrine filosofiche professate da Bernardo di Chartres, nessuno scritto a lui esplicitamente attribuito è stato rinvenuto fino ad oggi24. Bernardo è famoso soprattutto per aver inventato, o meglio aver rielaborato, la dottrina delle forme native, che sono la copia o l'immagine caduca, presente nella materia, delle idee intelligibili ed eterne; tale dottrina, insieme alle altre posizioni filosofiche di Bernardo riportate da Giovanni di Salisbury, in particolare la teoria dei paronimici, è stata oggetto di diversi studi nel corso del XX secolo ${ }^{25}$. Una ventina di anni fa, P. Dutton ha attribuito a Bernardo di Chartres un anonimo commento a lemmi sul Timeo conservato in diversi manoscritti, commento che era già stato studiato da T. Gregory negli anni Cinquanta ${ }^{26}$. Gli argomenti avanzati per tale attribuzione non risultano irrefutabili né pienamente convincenti: il commento «potrebbe» essere di Bernardo di Chartres.

Nella sezione intitolata dall'editore De constitutione mundi, commentando il lemma del Timeo 28A: Operi porro fortunam dat opifex suus, Bernardo di Chartres introduce la celeberrima distinzione calcidiana tra l'opus dei, l'opus naturae et l'opus artificis imitantis naturam ${ }^{27}$. Il mondo è l'opus dei e, in quanto tale, non ha né un principio né una fine nel tempo; le opera dei non sono temporalia ma causativa perché la loro causa è anteriore al tempo ed è conosciuta solo da dio:

24 É. Jeauneau ha ritrovato due versi che Giovanni di Salisbury attribuiva a Bernardo di Chartres in un manoscritto del Timeo conservato alla Biblioteca Vaticana: É. JEAunEAu, Gloses sur le «Timée» et Commentaire du «Timée» dans deux manuscrits du Vatican, «Revue des études augustiniennes», 8 (1962), pp. $365-373$.

25 É. Gilson, Le platonisme de Bernard de Chartres, «Revue néo-scolastique de philosophie», 25 (1923), pp. 5-19; T. Gregory, Note sul platonismo della scuola di Chartres. La dottrina delle «specie native», «Giornale critico della filosofia italiana», 8 (1953), pp. 358-362; ID., "Anima mundi». La filosofia di Guglielmo di Conches e la scuola di Chartres, Sansoni, Firenze 1955, pp. 76-79; J. JoLIvet, Quelques cas de platonisme grammatical du VIIe au XIIe siècle, in P. GALlaIs / Y.-J. Riou (éds.), Mélanges offerts à René Crozet, à l'occasion de son soixante-dixième anniversaire, I, Société d'Études Médiévales, Poitiers 1966, pp. 93-99; ID., Vues médiévales sur les paronymes, «Revue internationale de Philosophie», 113/3 (1975), pp. 222-242; ID., Rapports entre la grammaire et l'ontologie au Moyen Âge, in J.P. Beckmann (Hrsg.), Sprache und Erkenntnis im Mittelalter. Akten des VI. internationalen Kongresses für Mittelalterliche Philosophie der Société internationale pour l'étude de la philosophie médiévale (Bonn, 29. August-3. September 1977), de Gruyter, Berlin-New York 1981 («Miscellanea Medievalia», 13), pp. 135-164; ID., Platonisme et sémantique, de Bernard de Chartres aux Porretains, in C. Marmo (ed.), "Vestigia, Imagines, Verba». Semiotics and Logic in Medieval Theological Texts (XIIth-XIVth century). Acts of the XIth Symposium on Medieval Logic and Semantics (San Marino, 24-28 May 1994), Brepols, Turnhout 1997, pp. 9-17; P. Annala, The Function of the «formae nativae» in the Refinement Process of Matter: A Study of Bernard of Chartres's Concept of Matter, «Vivarium», 35/1 (1997), pp. 1-20; I. Caiazzo, Le glosse a Macrobio del codice Vaticano Latino 3874, «Archives d'histoire doctrinale et littéraire du Moyen Âge», 64 (1997), pp. 213-234; EAD., Lectures médiévales de Macrobe. Étude et édition des «Glosae Colonienses super Macrobium», Vrin, Paris 2002, pp. 132-141.

26 Gregory, Platonismo medievale cit., pp. 112-117.

${ }_{27} \mathrm{Su}$ Bernardo di Chartres, si veda anche il saggio di A. SpeER, Die entdeckte Natur: Untersuchungen zu Begründungsversuchen einer «scientia naturalis» im 12. Jahrhundert, Brill, Leiden-New York-Köln, 1995, pp. 93-103. 
«Est igitur mundus opus dei. Opera vero dei non sunt temporalia, quia nec principium nec finem habent in tempore. Vocantur quidem causativa, quia habent causas, ante tempus soli deo et non nobis cognitas, quae ita sunt fundamenta dei operum, sicut semina naturae operum naturalium, et ideo nihil patiuntur ex his quae infert tempus $[\ldots] \gg^{28}$.

Il mondo è detto eterno non nel senso che non ha una causa ma nel senso che è perpetuo e indissolubile; la sua causa, la volontà divina, è invece eterna ${ }^{29}$. Bernardo si fonda sul Commentarius di Calcidio dove, al capitolo 23, si legge che il mondo ha un'origo causativa, non temporaria ${ }^{30}$. In un altro luogo della sezione sulla costitutio mundi, commentando un passo famoso del Timeo: [...] omne visibile corporeumque motu importuno fluctuans neque umquam quiescens ex inordinata iactatione redegit in ordinem $[. .]^{31}$, Bernardo afferma che nella hyle, prima che venisse formata, vi era una certa confusione e che i semi dei corpi futuri giacevano fluttuando. La materia primordiale era dunque confusa e disordinata: ciò non suscita l'indignazione o la censura da parte di Bernardo di Chartres che commenta la lettera del Timeo senza distorcerne il significato, non cercando cioè di assimilare a tutti i costi il racconto timaico alla Genesi. Non vi è alcun tentativo evidente o esplicito di «cristianizzare» il Timeo:

«Redegit in ordinem omne visibile et corporeum, et hoc, dando proprias formas singulis. Fluctuans, id est in modum fluctuum se commiscens. Hoc ideo dicit, quia in hyle antequam formaretur, iactabatur seminarium corporum, non quod adhuc esset corpus, sed formandum erat, et ideo nitebatur ut formas acciperet. In qua hyle ipsa confusio erat, quasi fluctuatio et incerti motus. Illud vero seminarium nativis formis deus formavit, per quas discreta a se ipsis quattuor elementa, liquida et elimata, inventa sunt, nondum sensu comprehensibilia, et inde dicunt philosophi non ex nihilo deum fecisse mundum, sed tantum exornasse» 32 .

28 Bernardus Carnotensis, Glosae super Platonem 4.32-42 (Tim. 28A), ed. P.E. Dutton, Pontifical Institute of Medieval Studies, Toronto 1991 («Studies and Texts», 107), p. 158.

29 Bernardus Carnotensis, Glosae super Platonem 4.59-62, ed. Dutton, p. 159: «Propagatur etiam mundus aeternitati per causam, quia, cum voluntas dei mundi causa sit, quae eterna est, ipsa quoque eum aeternum facit. Nec dicimus aeternum quod careat principio, sed intelligimus perpetuum et indissolubilem».

${ }^{30}$ Calcidius, Commentarius 23, ed. Waszink, p. 74: «Et tempus immutationem aetatis morbos senectutem occasum invehit; his ergo omnibus quod a deo instituitur immune est origoque eius causativa est, non temporaria. Et mundus sensilis opus dei; origo igitur eius causativa, non temporaria. Sic mundus sensilis, licet et corporeus, a deo tamen factus atque institutus, aeternus est»; CALCIDIUS, Commentarius 25, ed. Waszink, p. 75: «Quid quod institutus est ad exemplum alterius intellegibilis et immutabilis perennitatis? Iam illud nemo dubitat, quae ad similitudinem instituuntur exempli sempiterni habere similitudinem perpetuitatis».

31 Tim. 30A, ed. Waszink, pp. 22-23.

32 Bernardus Carnotensis, Glosae super Platonem 4.185-195, ed. Dutton, p. 164. 
Infine, commentando il Timeo 31B, Bernardo di Chartres menziona i tre principi primi «platonici» che ha senz'altro rinvenuto nel Commentarius di Calcidio:

«[...]: est quippe unum deus, omnium opifex, alterum ideae, id est originales formae omnium quae numquam admiscentur creaturis, tercium hyle, materia scilicet corporum» 33 .

Nella sezione finale, intitolata dall'editore Tractatus de primordiali materia, Bernardo di Chartres focalizza la sua attenzione sulla natura della materia, ritornando su alcuni nodi dottrinali soltanto rapidamente dibattuti nelle precedenti sezioni. Bernardo afferma che fino a quel punto del dialogo - siamo a 47E - Platone ha parlato prevalentemente dei primi due principi, dio e le idee archetipe, e ha detto poco o nulla della hyle, il terzo principio, chiamata adesso in questa sezione necessitas, perché è impossibile che qualcosa di corporeo esista (esse) senza di essa, e questo sia se si sostiene che la hyle è la materia ex qua sono fatti i corpi sia se si sostiene che la hyle è la materia in qua sono fatti i cor$\mathrm{pi}^{34}$, cioè, si potrebbe glossare, sia se la materia è concepita in termini aristotelici sia se è concepita in termini platonici. Prima di proseguire nell'analisi della posizione abbastanza complessa di Bernardo di Chartres, è importante ricordare che nella traduzione calcidiana la materia è resa, quasi sempre, con necessitas e non si trova mai né materia né hyle né silva, vocaboli questi utilizzati da Calcidio nel Commentarius. Forse la composizione stessa dei manoscritti può aver avuto un'influenza sulla maniera in cui si leggeva il Timeo al tempo di Bernardo: fino alla metà del secolo XII, infatti, il Timeo è quasi sempre copiato insieme al Commentarius di Calcidio, in modo particolare il «primo» libro del Timeo (17A-39E) è seguito dal Commentarius (Praefatio, cc. 1-7 + I. Quae providentia dei contulerit, cc. 8-267) e il «secondo» libro del Timeo (39E-53C) è seguito dal Commentarius (II. Quae necessitas invexerit, cc. 268-355) ${ }^{35}$. Le edizioni moderne non rispettano queste divisioni «medievali» che possono risultare utili per render conto di alcune interpretazioni degli esegeti medievali del $\mathrm{Ti}^{\text {- }}$ meo. Ritornando a Bernardo di Chartres, egli preferisce dunque di gran lunga il termine hyle per designare la materia primordiale e non si serve mai di silva, con

33 Bernardus Carnotensis, Glosae super Platonem 4.235-237 (Tim. 31B), ed. Dutton, p. 166.

34 Bernardus Carnotensis, Glosae super Platonem 8.1-6, ed. Dutton, p. 218.

${ }_{35} \mathrm{Si}$ veda l'introduzione di WaszinK, De commentarii dispositione, pp. XVII-XXXv; cf. P.E. DutTon, Medieval Approaches to Calcidius, in G.J. ReYDAms-SchiLs (ed.), Plato's «Timaeus» as Cultural Icon, University of Notre Dame Press, Notre Dame 2003, pp. 183-205. Cf. anche l'introduzione di Jeauneau a GuILLELMUS de Conchis, Glosae super Platonem, Brepols, Turnhout 2006, pp. XLIX-L. 
una sola eccezione ${ }^{36}$, termine che adotterà invece, come è noto, Bernardo Silvestre nella Cosmographia. Come interpretare questa scelta di Bernardo di Chartres? De silva è il titolo che Calcidio ha dato al capitolo sulla materia e il vocabolo silva è utilizzato in lungo e in largo nella trattazione sulla materia che occupa tutta la seconda parte del Commentarius, cc. 268-355.

Bernardo di Chartres spiega che i sostenitori della teoria che la materia primordiale sia solamente ciò in quo fiunt i corpi pensano quindi che la hyle non sia una delle cause principali (alla stregua cioè di dio e delle idee archetipe) della formazione dei corpi ma soltanto il luogo in cui arrivano le forme native che generano i corpi; le forme native fanno dunque le veci del padre mentre la hyle funge da ventre della madre che riceve il seme del padre ${ }^{37}$. Ma allora - replica in una sorta di disputatio immaginaria Bernardo ai sostenitori dell'in quo - com'è possibile che qualcosa possa nascere (ex qua fiat aliquid) da una hyle concepita in siffatta maniera visto che essa non ha la capacità di generare i corpi ma soltanto di ricevere le forme native? Ebbene - continua Bernardo -, secondo costoro, vi sarebbe una materia confusa creata ex nihilo oppure senza origine (originem carens) che, una volta ricevute le forme native, si trasformerebbe negli elementi purificati (eliquata elementa); successivamente, la sovrapposizione di ulteriori forme native consentirebbe agli elementi puri di trasformarsi in elementaria, e così di seguito. L'inizio della trattazione sulla materia primordiale riprende quindi un tema già discusso in maniera più sintetica nella sezione sulla constitutio mundi ${ }^{38}$. Ma risulta evidente che questa teoria della hyle come un in quo non piace a Bernardo di Chartres: i corpi vengono fatti ex hyle e non in hyle, anche se a volte si dice che qualcosa è fatto nella materia, come quando si dice che la statua è fatta «nella cera». La hyle è quasi pater in quanto, una volta ricevute le forme native, si trasforma in corpo, quasi mater in quanto riceve in sé le forme. Poiché svolge il ruolo di pater, la hyle è la causa principalis dei corpi dopo dio e le idee archetipe, mentre in quanto adempie il ruolo di mater la hyle è una causa secundaria:

«Erit igitur hyle quasi pater, secundum hoc quod assumptis formis transit in corpus; quasi mater, secundum hoc quod in se recipit formas. Et secundum quod pater, prin-

36 Bernardus Carnotensis, Glosae super Platonem 8.366, ed. Dutton, p. 231: «Nota hylen, id est silvam, nec intelligibile $[\ldots] »$.

${ }^{37}$ Bernardus Carnotensis, Glosae super Platonem 8.6-9, ed. Dutton, p. 218: «Dicitur enim a quibusdam hylen non esse principalem causam corporum, sed solas nativas formas quae obtinent [obtinet $e d$. Dutton, ma un manoscritto comporta la lezione obtinent che io preferisco] vicem patris, quia sicut semen patris in alvo matris efficit filium, ita formae nativae venientes in hylen generant corpora. Ipsa vero hyle vicem obtinens matris necessaria fuit ad mundi generationem, ideoque dicitur necessitas».

38 Vedi sopra, p. 252 e n. 32. 
cipalis causa erit corporum post deum et ideas; secundum quod mater, secundaria causa»39.

Per Bernardo di Chartres, dunque, la hyle è passiva e attiva al tempo stesso; la soluzione platonica, cioè la materia intesa come un in quo, come mero ricettacolo, non gli sembra sufficiente per spiegare in modo soddisfacente la produzione dei corpi e, soprattutto, egli vuole a tutti i costi mantenere sullo stesso piano le tre cause o principi platonici: dio, idee archetipe e materia. Bernardo concepisce dunque la materia in termini parzialmente aristotelici (come Calcidio, prima di lui, del resto): la hyle è al tempo stesso l'in quo in cui vengono prodotti i corpi e l'ex quo che produce i corpi. Le due teorie coesistono e sono entrambe utilizzate, secondo le sue esigenze ermeneutiche, in questa parte finale del commento $^{40}$. Ma risulta evidente che il perno di tutto il sistema metafisico di Bernardo di Chartres sono le forme native, imagines idearum ${ }^{41}$ che scendono nella materia e la trasformano — quei simulacra idearum di cui già parlava Platone nel Timeo 50C che «entrano ed escono dalla materia in una maniera difficile da spiegare» 42 . In un altro luogo del commento, Bernardo precisa poi che prima della costituzione del mondo tutte le forme native, che sarebbero arrivate nella hyle successivamente, erano già presenti potentialiter nella stessa hyle, mentre le forme dei futuri quattro elementi erano già presenti actualiter nella hy$l e$, quasi a voler sottolineare la priorità ontologica dei quattro elementi rispetto a tutti gli altri corpi risultanti dal congiungimento delle forme native e della $h y-$ le. I principi sono comunque sempre e solo tre, cioè dio, la hyle e le idee:

«Et nota quoniam, licet ante constitutionem mundi omnes nativae formae quae post in hylen venerunt in ipsa hyle tantum potentialiter exstiterunt, illae tamen quae ipsam ad quattuor mundi elementa procreanda formabant, actualiter ante mundi exornationem in ipsa constiterunt; non tamen ut carentes origine, ne sint plura principia prima quam tria: scilicet deus, hyle et ideae» ${ }^{43}$.

La materia primordiale (hyle nel vocabolario di Bernardo) è chiamata con diversi nomi nel Timeo, nomi che Bernardo cerca di spiegare, servendosi soprat-

39 Bernardus Carnotensis, Glosae super Platonem 4.19-22, ed. Dutton, pp. 218-219.

40 Per esempio, Bernardus Carnotensis, Glosae super Platonem 8.73 (Tim. 48C), ed. Dutton, p. 221: «Quia dicturus erat quod illa quattuor pura elementa ex hyle vel in hyle fierent [...]».

41 Bernardus Carnotensis, Glosae super Platonem 8.201-202 (Tim. 50B), ed. Dutton, p. 226.

42 Tim. 50C, ed. Waszink, p. 48: «Quae vero ingrediuntur, formas mutant aliasque alia et diversa cernuntur, eademque quae introeunt et egrediuntur simulacra sunt vere existentium rerum miro quodam vixque explicabili modo formata ab isdem vere existentibus rebus, quem ad modum mox demonstrare nitemur pro viribus».

43 Bernardus Carnotensis, Glosae super Platonem 8.397-402, ed. Dutton, p. 232. 
tutto del Commentarius di Calcidio: prima di tutto, necessitas ${ }^{44}$, il termine discusso all'inizio del Tractatus de primordiali materia; in seguito, la causa errante «erraticam causam vocat hylen, quia in ea ante informationem hanc et discretionem omnia quasi fluctuando errabant»45, e secondo questa accezione, puntualizza Bernardo, la hyle non è la causa principale del mondo sensibile, cioè questa appellazione mette l'accento sulla connotazione spaziale, sull'in quo, della hyle ${ }^{46}$; la hyle è detta fraudes perché induce in errore i sensi e la ragione ${ }^{47}$; la hyle è pure receptaculum e nutricula perché nutre le forme che riceve ${ }^{48}$; la hyle è mater omnium corporum ${ }^{49}$; la hyle è locus perché in essa scendono le forme native ${ }^{50}$. Un altro punto estremamente importante nel commento di Bernardo è una lunga discussione sulla natura della hyle - quid sit, substantia scilicet an accidens? - inserita durante l'elucidazione del Timeo 51B: Si forte in se recipit simulacra etc ${ }^{51}$. Questa stessa discussione, con qualche variante minima senza importanza, si ritrova nelle Glosae Colonienses super Macrobium (12,7: haec est yle), commento anonimo sui Commentarii in Somnium Scipionis composto, molto probabilmente, all'inizio del secolo XII52.

Bernardo di Chartres ha illustrato il Timeo cercando di comprenderne pienamente la lettera e scegliendo di farsi guidare dal Commentarius di Calcidio; in particolar modo, non ha cercato di «battezzare Platone». Se nel suo commento è possibile pure rintracciare accenni rapidi al cristianesimo e alla Trinità, essi rimangono comunque estremamente discreti. Non è probabilmente esatto inscrivere questo commento nella corrente di pensiero «chartriana» che cerca per l'appunto di far convergere la filosofia pagana e il dogma cristiano (si pensi ad autori come Guglielmo di Conches e Teodorico di Chartres). Sarebbe allora più

44 Vedi sopra p. 246.

45 Bernardus Carnotensis, Glosae super Platonem 8.39-40, ed. Dutton, p. 219.

46 Bernardus Carnotensis, Glosae super Platonem 8.50-52, ed. Dutton, p. 220: «Nota etiam, secundum quod dicitur erratica causa, ipsam hylen videri non esse principalem causam huius mundi, quod concedimus, secundum quod supra determinatum est».

47 Bernardus Carnotensis, Glosae super Platonem 8.116-120, ed. Dutton, p. 222: «Hylen ideo vocat fraudem, quia nec sensu concipi secundum se nec vera ratione potest. Sensus enim non credit rem incorpoream, cum videat eam coloratam. Ratio non credit eam corpoream, cum secundum propriam naturam nullam in ea inveniat corporum qualitatem».

48 Bernardus Carnotensis, Glosae super Platonem 8.127-130, ed. Dutton, pp. 222-223: «Quod sit receptaculum et nutricula verum est. Hic haerent qui dicunt hylen esse materiam in qua, non ex qua, fiant corpora, sed frustra. Rectius enim aes dicitur nutrire formas quas recipit, ut fiat statua, vel etiam ipsam statuam, quam id in quo fabricatur statua. Sic et hyle».

49 Bernardus Carnotensis, Glosae super Platonem 8.234, ed. Dutton, p. 227.

50 Bernardus Carnotensis, Glosae super Platonem 8.354-355, ed. Dutton, p. 231.

51 Bernardus Carnotensis, Glosae super Platonem 8.264-310, ed. Dutton, pp. 228-229.

52 Cf. Cainzzo, Lectures médiévales de Macrobe cit., pp. 211-213. Vi sono altri luoghi paralleli tra le Glosae super Platonem di Bernardo di Chartres e le Glosae Colonienses super Macrobium. 
giusto collocare questo commento agli inizi di una tradizione esegetica del $T i$ meo che abbia eventualmente preceduto il movimento «chartriano»?

\section{Le Glosae super Platonem di Guglielmo di Conches}

Grammaticus post Bernardum opulentissimus: così Giovanni di Salisbury definisce Guglielmo di Conches nel Metalogicon ${ }^{53}$. Della vita e della carriera di questo autore, nato forse verso il 1080 e morto verso il 1154, si sa poco o nulla, e una trentina di anni fa R. Southern ha addirittura rimesso in discussione il fatto che fosse un maestro della scuola cattedrale di Chartres, sollevando una serie di proteste e provocando un animato dibattito tra gli studiosi del secolo XII ${ }^{54}$. Guglielmo di Conches è l'autore di varie opere: due trattati, la Philosophia e il Dragmaticon; diversi commenti a lemmi su testi classici e tardo-antichi: la Consolatio Philosophiae di Boezio, i Commentarii in Somnium Scipionis di Macrobio, il Timeo di Platone, le Institutiones grammaticae di Prisciano e, probabilmente, le Satirae di Giovenale e il De nuptiis Mercurii et Philologiae di Marziano Capella ${ }^{55}$. Se la cronologia relativa delle opere di Guglielmo sembra oramai sicura, le date di composizione rimangono congetturali ma in ogni modo abbastanza affidabili; le Glosae super Platonem occupano una posizione centrale: redatte quasi sicuramente verso il 1130, esse seguono la Philosophia (verso il 1125) e precedono il Dragmaticon (1144-1147).

Le Glosae super Platonem sono un'opera ampia e complessa: diversi passaggi offrono spunti interessanti per la riflessione sulla materia primordiale, sulla sua relazione con dio e con i quattro elementi. Guglielmo di Conches riprende la tripartizione calcidiana dell'opus proprio di dio, della natura e dell'artigiano, che diventa sotto la sua penna opus creatoris (e non dei, come in Bernardo, o opificis, come in Calcidio), opus naturae e opus artificis imitantis naturam - distinzione questa inserita durante la spiegazione del lemma del Timeo 28A: Operi

53 IoAnnes SAREsberiensis, Metalogicon I, 5, ed. Hall / Keats-Rohan, p. 20.

54 R. Southern, Medieval Humanism and Other Essays, Harper \& Row, New York 1970 / Blackwell, Oxford 19842; ID., Platonism, Scholastic Method, and the School of Chartres: the Stenton Lecture 1978, Reading University Press, Reading 1979; ID., Scholastic Humanism and the Unification of Europe, I, Blackwell Publishing, Oxford-Cambridge 1995. In quest'ultimo saggio R. Southern tempera molto le sue critiche. Per le risposte a R. Southern, cf. P. Dronke, New Approaches to the School of Chartres, "Anuario de estudios medievales», 6 (1969), pp. 117-140; N. Häring, Chartres and Paris Revisited, in J.R. O'DonNELL (ed.), Essays in Honour of A.C. Pegis, Pontifical Institute of Medieval Studies, Toronto 1974, pp. 268329.

55 Si veda l'introduzione di Jeauneau alla nuova edizione delle Glosae super Platonem, CCCM 203, Brepols, Turnhout 2006. Tutte le citazioni inserite nel presente studio provengono da tale edizione; si segnala che la numerazione dei capitoli è invariata rispetto all'edizione precedente: GUILLAUME DE CONCHES, Glosae super Platonem, ed. É. Jeauneau, Vrin, Paris 1965. 
porro formam dat opifex suus ${ }^{56}$. Per Guglielmo, l'opus creatoris è la "prima creatio sine preiacente materia, ut est creatio elementorum, et spirituum, vel ea que videmus fieri contra consuetum cursum naturae, ut partus virginis, etcetera» 57 . Il creator non ha dunque bisogno di una materia preesistente per creare i quattro elementi e gli spiriti ed è inoltre responsabile degli eventi miracolosi che sembrano andare contro il consueto corso della natura. Lopifex platonico (calcidiano) è per Guglielmo di Conches il dio creatore dei cristiani, come afferma esplicitamente e senza equivoci commentando pure, per esempio, il Timeo 30D: «Ergo opifex, id est creator», senza dimenticare che egli nega categoricamente l'esistenza di un caos primordiale anteriore all'azione del creatore, che giudica inutile e contrario ad ogni logica58. Inoltre, l'opus trae le sue qualità e le sue caratteristiche dall'artigiano che l'ha realizzato e perciò l'opus creatoris «perpetuum est, carens dissolutione: neque enim mundus neque spiritus dissolvuntur» ${ }^{59}$; il mondo sensibile è perfetto e contiene solo il bene ${ }^{60}$. Guglielmo è il primo commentatore del Timeo nel secolo XII ad affermare senza ambiguità che il dio platonico è il dio dei cristiani, adottando come linea interpretativa degli scritti degli antichi la cosiddetta rivelazione naturale: cioè Platone e altri grandi saggi e profeti dell'Antichità hanno avuto una prefigurazione della rivelazione cristiana che hanno cercato di esprimere nei loro scritti, anche se in modo

56 Guillelmus de Conchis, Glosae super Platonem 37, ed. Jeauneau, p. 70. Guglielmo commenta il lemma sotto questa forma, mentre nell'edizione di Waszink si legge: Operi porro fortunam dat opifex suus. $\grave{E}$ interessante notare che Bernardo di Chartres commenta il lemma fortuna e non forma. Bisogna allora dedurne che Guglielmo di Conches e Bernardo di Chartres non hanno utilizzato un testimone appartenente alla stessa tradizione manoscritta del Timeo? Jeauneau ha segnalato altri lemmi «discordanti» tra questi due autori che, appartenendo alla stessa scuola di Chartres, dovrebbero, teoricamente, utilizzare la stessa tradizione manoscritta del Timeo.

57 Guillelmus de Conchis, Glosae super Platonem 37, ed. Jeauneau, p. 69.

58 Guillelmus de Conchis, Glosae super Platonem 56, ed. Jeauneau, p. 99; creator è utilizzato in molti luoghi delle Glosae, cf. per esempio, 43, 44, 48, ed. Jeauneau, pp. 78, 79, 86. Sulla questione del caos primordiale in Guglielmo di Conches si possono consultare: M.-D. Chenu, Nature ou histoire? Une controverse exégétique sur la crétion au XIIe siècle, «Archives d'histoire doctrinale et littéraire du Moyen Âge», 28 (1953), pp. 25-30; É. Jeauneau, Du désordre à l'ordre (Timée 30A), in T. LeinKauf / C. Steel (eds.), Platons «Timaios» als Grundtext der Kosmologie in Spätantike, Mittelalter und Renaissance. Plato's «Timaeus» and the Foundations of Cosmology in Late Antiquity, the Middle Ages and Renaissance, Leuven University Press, Leuven 2005 («Ancient and Medieval Philosophy», 34), pp. 253-263; H. MERLE, «Sic dissolutum est Chaos». Monographie sur le mythe et la notion de Chaos, héritage de l'Antiquité au Moyen Âge, in A. Cazenave / J.F. Lyotard (éds.), L'art des confins. Mélanges offerts à Maurice de Gandillac, Presses Universitaires de France, Paris 1985, pp. 365-386; S. VIARRE, Cosmologie antique et commentaire de la création du monde. Le chaos et les quatre éléments chez quelques auteurs du Haut Moyen Âge, in AA.Vv., La cultura antica nell'Occidente latino dal VII all'XI secolo. Atti della XXII Settimana di studio (Spoleto, 18-24 aprile 1974), Centro Italiano di Studi sull'Alto Medioevo, Spoleto 1975, pp. 541-573; G. GarfaGNINI, Cosmologie medievali, Loescher, Torino 1978.

59 Guillelmus de Conchis, Glosae super Platonem 37, ed. Jeauneau, p. 69.

60 Guillelmus de Conchis, Glosae super Platonem 38, ed. Jeauneau, p. 71. 
imperfetto perché si sono serviti di espressioni non sempre conformi al dogma cristiano. Invece, il contemporaneo Ugo di San Vittore, nel commento alla $G e$ nesi, traendo ispirazione dalla ricca e cospicua tradizione alto-medievale dei commenti genesiaci, spiega che i filosofi, in realtà Platone e i suoi seguaci, e gli autori cristiani la pensano diversamente relativamente ai tre principi primi:

«In hoc enim differunt auctores nostri a philosophis, quod philosophi deum opificem tantum, et tria ponunt principia: deum, materiam et archetipas ideas; nostri vero unicum ponunt principium, et hoc deum solum» ${ }^{61}$.

Un riferimento a delle glosse sulla Genesi si legge nell'inedito Apparatus super Thimeum, un commento a lemmi anonimo sul Timeo redatto nella seconda metà del secolo XII; secondo l'autore, i tre nomi impiegati da Platone per i tre principi primi coeterni non sono altro che un modus loquendi per designare lo stesso identico principio:

«Imago alterius (Tim. 29A). [...] Plato enim, sicut in principio glosarum super Genesim legitur, tria universitatis rerum principia coeterna ponebat: deum, materiam quae yle, exemplar quod archetipus mundus dicitur. Deum autem ponebat quasi efficientem, ylem quasi materialem, mundum archetipum quasi exemplarem causam mundi; et haec dicebat tria esse coeterna universitatis rerum principia. [...] Unde plura rerum principia eterna ut ponebat Plato putare non debemus, licet nomina ipsa plura, plura sonare videntur principia. Nomina enim haec deus, hyle, mundus archetipus quasi tria diversa significant, plenius tamen considerati nichil esse quid [...] hoc nomine yle, nichil esse quod appellatur mundus archetipus; patebat tamen nomina illa esse inventa ad diversos quosdam loquendi modos designandos quos ad si facilem et ad planum loquendi modum reducamus, facile patebit veritas» 62 .

$\mathrm{Al}$ contrario, e questo va sottolineato perché è estremamente importante, $\mathrm{Gu}$ glielmo di Conches non parla nelle Glosae super Platonem dei tre principi primi di calcidiana memoria e ripresi, come si è visto, da Bernardo di Chartres; il motivo è che per lui la materia è creata, dunque creatura, e le idee archetipe sono nella mente divina. Il ruolo metafisico della materia primordiale è allora notevolmente ridimensionato perché essa dipende ontologicamente in tutto e per tutto dal dio creatore.

I primi lemmi del Timeo citati nel Tractatus de primordiali materia, la sezione finale delle Glosae super Platonem, introducono un lungo excursus sulla ma-

${ }^{61}$ Hugo de Sancto Victore, In Genesim 4, PL 175, 33. Si veda Gregory, Anima mundi cit., pp. 4748 per altre testimonianze: Pietro Lombardo, Ugo di Rouen, Pietro Mangiadore.

$62 \mathrm{Ms}$ Salamanca, Biblioteca Universitaria, 2322, f. 167rA-B. Su questo commento si vedano i lavori di DutTon e Caiazzo citati alla nota 22. 
teria; Guglielmo di Conches fornisce in primo luogo una definizione generale della materia - «materia igitur est quod, accepta forma, transit in aliud ut aes in statua» 63 - per poi precisare che esiste una materia prima e una materia secon$\mathrm{da}$, riprendendo molto probabilmente una distinzione che si legge nel Commentarius di Calcidio in cui la silva è detta subiectio principalis (sostrato principale) ed è distinta dalla materia «materiale» (bronzo, legno) sulla quale opera invece l'artigiano ${ }^{64}$ : «Prima materia est quae ita est materia quod nichil est eiusdem materia, secundaria quae ita est materia quod aliquid est eiusdem materia»65. Questa definizione della materia prima riceve il consenso generale, ma i dissensi sorgono - scrive Guglielmo - laddove si intenda riferire tale definizione a un qualcosa di preciso. Alcuni ritengono che la materia prima non sia altro che $\mathrm{i}$ quattro elementi nel caos primordiale, ma questa tesi è subito esclusa: la materia primordiale non è i quattro elementi, sia che essi si trovino nel caos sia fuori dal caos, perché i quattro elementi sono dotati di una forma - il fuoco è acuto, la terra ottusa, ecc. - e di qualità proprie - il fuoco è caldo e secco, ecc. Ma perfino nel caos primordiale i quattro elementi dovevano possedere già le stesse qualità sostanziali che si riscontrano fuori dal caos, qualità senza le quali nessuna cosa può veramente sussistere; la materia primordiale, viceversa, non ha né forma né qualità ${ }^{6}$. Allora la materia dei quattro elementi è la materia prima ricercata, come dimostra abilmente Guglielmo in questo paragrafo:

«Est ergo nostra sententia quod materia elementorum est prima materia. Quod materia sit, nulla dubitatio est. Quod prima est, probatur quia nichil est eius materia. Si enim esset aliquid illius materia, esset eiusdem et forma, quia quidquid habet formam, habet et materiam: esset ergo materia elementorum quiddam constans ex materia et forma, et sic esset corpus. Sed si esset corpus, esset elementum vel factum ex elementis. Sed neque elementum neque factum ex elementis est materia elementorum. Est ergo materia elementorum talis materia cuius nichil est materia: est ergo prima materia» 67 .

Se la materia primordiale è la materia dei quattro elementi, i quattro elementi sono, a loro volta, la materia del mondo, come Guglielmo aveva già indicato nella sezione dedicata alla generazione del mondo sensibile68. La materia primordiale non esiste per se ma si può solo intelligere per se poiché «vis est intellectus

63 Guillelmus de Conchis, Glosae super Platonem 154, ed. Jeauneau, 277. Si vedano anche le osservazioni di Gregory, «Anima mundi» cit., pp. 193-195.

64 Calcidius, Commentarius 337, ed. Waszink, p. 330.

65 Guillelmus de Conchis, Glosae super Platonem 154, ed. Jeauneau, p. 277.

66 Guillelmus de Conchis, Glosae super Platonem 154, ed. Jeauneau, p. 278.

67 Guillelmus de Conchis, Glosae super Platonem 154, ed. Jeauneau, pp. 278-279.

68 Guillelmus de Conchis, Glosae super Platonem 63, ed. Jeauneau, p. 114: «Postquam materiam mun- 
coniuncta disiungere et disiuncta coniungere»69. Questa materia che non può essere percepita attraverso i sensi viene dunque compresa (intelligitur) grazie all'intelletto astraendo dalle cose, cogitatione etsi non actu, le qualità e le forme: secondo Calcidio - scrive Guglielmo -, così come le tenebre si vedono non vedendo e il silenzio si ascolta non ascoltando, la materia primordiale intelligitur non intelligendo ${ }^{70}$. La materia primordiale non è né una sostanza né un accidente ma, come dice Platone, essa è inter nullam et aliquam substantiam; la materia è una creatura e non un creatore, perché dio poté creare simultaneamente e gli elementi e la materia degli elementi («fuit enim deus tantae potentiae quod simul elementa et eorum materia creare potuit» $)^{71}$. Tale creazione avvenne fuori del tempo nella mente divina, perché «Qui manet in aeternum creavit omnia simul» - scrive Guglielmo di Conches citando l'Ecclesiasticus (Sir.) 18, 1; questo versetto biblico è frequentemente citato e variamente interpretato dagli autori del secolo XII 72 . Guglielmo di Conches si distacca dall'interpretazione calcidiana del Timeo sostenendo che la materia è creata da dio; diversamente, Bernardo di Chartres, rispettando la lettera del racconto timaico e influenzato da Calcidio, non aveva affermato apertamente che la materia era stata creata da dio. La materia dunque per Guglielmo non è un principio primo eterno e nel Timeo essa è detta necessitas semplicemente perché non può esistere senza i corpi né $\mathrm{i}$ corpi possono esistere senza di essa ${ }^{73}$. Per tentare allora di comprendere la vera natura della materia, è necessario parlare dei quattro elementi e delle loro reciproche mutazioni. Infatti, quando gli elementi si trasformano l'uno nell'altro, le variazioni delle loro qualità si producono nella materia che sola può subire $\mathrm{i}$ cambiamenti e accogliere le qualità: la hyle è la materia comune degli elemen-

di, scilicet quatuor elementa, ostendit, et quare quatuor nec pauciora facta sunt, subiungit qualiter ex tali materia factus est mundus, ita scilicet quod nichil de illis extra mundum relictum est».

69 Guillelmus de Conchis, Glosae super Platonem 154, ed. Jeauneau, p. 279. La citazione «vis est intellectus...», ricorrente negli scritti di Guglielmo di Conches, proviene da BoETus, In Porphyrii Isagogen commentorum editio secunda I, 2, ed. S. Brandt, CsEL 48, Wien 1906, p. 165.

70 Guillelmus de Conchis, Glosae super Platonem 155, ed. Jeauneau, pp. 279-280: «Sed dicit inde Calcidius quod sicut tenebrae videntur non videndo et silentium auditur nichil audiendo, sic hyle, illa primordialis materia, intelligitur non intelligendo. Quod est dicere: quemadmodum oportet visum deficere et tenebrae videantur et auditum similiter ut silentium audiatur, ita et intellectum ut hyle intelligatur. Cum enim materiam sine omni forma cogitamus, noster deficit intellectus».

71 Guillelmus de Conchis, Glosae super Platonem 155, ed. Jeauneau, pp. 280-281.

72 Guillelmus de Conchis, Glosae super Platonem 156, ed. Jeauneau, p. 281. Si veda Parent, La doctrine de la Création cit.; C. Gross, Twelfth-Century Concepts on Time: Three Reinterpretations of Augustine's Doctrine of Creation «Simul», «Journal of History of Philosophy», 23 (1985), pp. 325-338; R.C. DALES, Discussions of the Eternity of the World during the First Half of the Twelfth Century, «Speculum», 57 (1982), pp. 495-508; ID., Medieval Discussions of the Eternity of the World, Brill, Leiden-New York-København-Köln 1990 («Brill's Studies in Intellectual History», 18).

73 Guillelmus de Conchis, Glosae super Platonem 156, ed. Jeauneau, p. 282: «necessitas id est indigentia, quia nec potest existere sine corporibus nec corpora sine ipsa». 
ti capace di ricevere e gli elementi e le loro qualità ${ }^{74}$. Dopo un lungo excursus sui quattro elementi, Guglielmo, glossando il Timeo 49A, parla nuovamente della natura e della forza della hyle:

«Natura igitur hyles est quod careat omni forma et omni qualitate. Cum enim sit materia communis omnium elementorum, si aliquam haberet qualitatem vel formam similem alicui illorum, citius transiret in illud cui similis esset et sic dissolverentur cetera elementa. Vis vero illius est quod quamvis nullam habeat formam vel qualitatem propriam, omnium tamen formas et qualitates potest recipere, ut omnia ex ipsa possint constare» 75 .

È interessante segnalare che, glossando il Timeo 49E, Guglielmo di Conches ne approfitta per inserire alcune considerazioni di ordine grammaticale che devono essere poste in relazione con questioni dibattute in un'altra sua opera, le Glosae super Priscianum. Il pronome può indicare soltanto la materia primordiale - scrive Guglielmo - cosicché alcuni (quidam) per spiegare il luogo delle Institutiones di Prisciano, pronomen significat meram substantiam ${ }^{76}$, glossano «id est primordialem materiam quae caret omni forma et omni qualitate»77. Il nome infatti, che significa la sostanza e le qualità stabili, non può nominare la materia primordiale «propter mutabilitatem qualitatum circa ipsam» 78 .

Guglielmo di Conches ripete in più occasioni che la materia primordiale non ha né forma né qualità perché, se avesse una forma propria, l'arrivo di un'altra forma creerebbe un turbamento ${ }^{79}$; essa è però capace di ricevere tut-

74 Guillelmus de Conchis, Glosae super Platonem 158, ed. Jeauneau, pp. 285-286: «Has perpessiones potius considerat, quia per istas, ut ait Calcidus, aperte intelligitur hyle. Cum enim terra in aquam mutatur vel aqua in terram, variantur qualitates. Sed omnis variatio qualitatum fit circa aliquam materiam quae potest illas suscipere. Ergo in hac mutatione terrae et aquae oportet aliquam materiam esse quae qualitates terrae et aquae possit recipere. Sed hoc non potest esse terra: est enim una qualitas aquae, scilicet humiditas, quae non potest inesse terrae. Similiter non potest esse aqua nec aliud elementum. Ergo perpendit ratio in mutabilitate elementorum esse quandam materiam communem istis quae huius et illius qualitates in se possit recipere. Et hoc est hyle».

75 Guillelmus de Conchis, Glosae super Platonem 161, ed. Jeauneau, p. 292.

76 Priscianus Caesariensis, Institutiones grammaticae XII, 1, ed. H. Keil, GL II, Leipzig 1855, p. 577. Guglielmo commenta questo luogo nelle Glosae super Priscianum, Ms Firenze, Biblioteca Medicea Laurenziana, San Marco 310, f. 59vB (prima redazione) e Ms Parigi, BnF, lat. 15130, f. 76vA (seconda redazione). Per la discussione di questo passo nelle Glosae super Priscianum, si veda l'articolo di K.M. FrEDBorg, The dependance of Petrus Helias" "Summa super Priscianum» on William of Conches" "Glosae super Priscianum», «Cahiers de l'Institut du Moyen Âge grec et latin», 11 (1973), pp. 1-57 (in particolare pp. 46-50). Nella seconda redazione delle Glosae super Priscianum, Guglielmo non approva quegli autori che affermano che il pronome indica la mera substantia, assumendo quindi una posizione diversa rispetto alle Glosae super Platonem. Questo punto meriterebbe un approfondimento ulteriore.

77 Guillelmus de Conchis, Glosae super Platonem 164, ed. Jeauneau, p. 299.

78 Guillelmus de Conchis, Glosae super Platonem 165, ed. Jeauneau, p. 300.

79 Guillelmus de Conchis, Glosae super Platonem 167, ed. Jeauneau, p. 304: «Si enim haberet propriam formam, cum alia superveniret, una turbaret aliam». 
te le forme ${ }^{80}$. La hyle è detta anche «ricettacolo» perché riceve le forme e le qualità di tutte le cose che nascono, e «nutrice» perché tutti i corpi in essa contenuti aumentano. Tuttavia dio non continua a creare della materia nuova, ma nella materia creata, insieme agli stessi elementi, le forme e le qualità subiscono delle variazioni; allo stesso modo, nessuna materia è annientata o distrutta, e le forme e le qualità che si distaccano dai corpi si risolvono nei quattro elementi:

«Hyle est receptaculum omnium quae gignuntur, quia formas et qualitates eorum in se recipit. Est quasi nutricula, quia circa ipsam omnia corpora augmentantur. Credimus enim nullam materiam novam a deo creari, sed circa materiam cum ipsis elementis creatam formas et qualitates variari. Et quemadmodum nullam credimus novam gigni, credimus nullam materiam adnichilari, sed formas et qualitates amittere et ad elementa resolvi» 81 .

Riprendendo un lemma già citato all'inizio del trattato sulla materia primordiale, inter nullam et aliquam substantiam (Tim. 51A), Guglielmo spiega che la materia «nec penitus est nulla nec penitus est aliqua» perché in quanto materia di altre cose non è nulla sostanza, ma non è nemmeno una aliqua sostanza perché non esiste di per sé. Ed è per questo motivo che Calcidio afferma che bisogna chiamarla «aliqua nulla» e «nulla aliqua». Esiste anche un'altra interpretazione possibile: con nulla substantia si indicano i sensibili che sono soggetti al mutamento in permanenza e con aliqua substantia gli intelligibili, immutabili, come il mondo archetipo e le idee in esso contenute; la materia dunque gode di uno statuto intermedio tra i sensibili e gli intelligibili perché essa non è né veramente sensibile né veramente intelligibile:

«Vel: nulla substantia dicantur ista sensibilia quia non subsistunt in eodem statu sed semper mutantur; aliqua substantia dicantur intelligibilia, ut archetipus mundus et ideae quae in eo sunt, propter immutabilitatem sui. Est ergo hyle inter nullam et aliquam substantiam, id est inter sensibilia et intelligibilia, quia nec perfecte est sensilis, nec perfecte est intelligibilis» 82 .

La materia non è intelligibile perché viene compresa defectu intellectus, cioè è intelletta senza intelligere, come ha già spiegato sopra, ma non è nemmeno sensibile perché non si può sentire perfettamente; essa si può percepire soltanto at-

80 Guillelmus de Conchis, Glosae super Platonem 169, ed. Jeauneau, p. 308.

81 Guillelmus de Conchis, Glosae super Platonem 161, ed. Jeauneau, p. 293; Guillelmus de Conchis, Glosae super Platonem 173, ed. Jeauneau, p. 315: «(In Tim. 52B) numquam materia adnichilatur sed formae et qualitates circa eam variantur».

82 Guillelmus de Conchis, Glosae super Platonem 169, ed. Jeauneau, p. 308. 
traverso i suoi effetti, cioè attraverso le mutazioni e le trasformazioni dei quattro elementi o meglio attraverso le trasformazioni delle qualità e delle forme proprie ai quattro elementi83. Guglielmo di Conches mette dunque l'accento sulle caratteristiche «fisiche» della materia, sul fatto che essa è il sostrato e il luogo («genus loci quia in se omnem formam comprehendit») 84 in cui avvengono le trasmutazioni dei quattro elementi, e non tanto sulla sua potenzialità, sulla sua capacità di produrre i corpi sensibili: in breve, si può dire che Guglielmo propende per una materia concepita come un in quo piuttosto che come un ex quo.

83 Guillelmus de Conchis, Glosae super Platonem 169, ed. Jeauneau, p. 308; Guillelmus de Conchis, Glosae super Platonem 174, ed. Jeauneau, p. 317; GullLelmus De Conchis, Glosae super Platonem 162, ed. Jeauneau, pp. 294-295. Si veda I. Cainzzo, Les quatre éléments dans l'œuvre de Guillaume de Conches, in I. Caiazzo / B. OвRist (éds.), Guillaume de Conches: philosophie et science au XIIe siècle, Brepols, Turnhout, in corso di stampa.

84 Guillelmus de Conchis, Glosae super Platonem 173, ed. Jeauneau, p. 315. 